A total of 48 patients were identified from 20 publications (51 including our patients). Indications for insertion included extensive deep vein thrombosis (DVT) (39), PE (7), recurrent thrombosis (1), contraindication to anticoagulation (1) and unknown (3). Complications at insertion occurred in 2 patients. Two philtres were not removed, one philtre was repositioned and outcome was unclear in 5 . Therefore $37 / 40$ were retrieved successfully (81\%). Retrieval failed in 8 women because of failure to snare (2), tilting (4), fracture and migration of the device(1) and occlusion of the philtre with thrombus (1).

The incidence of failed retrieval of IVC philtres in pregnancy $(18 \%)$ is at the higher end of the spectrum reported in the general population $(0-22 \%)$. Careful consideration of the benefits and risks of IVC philtre placement in pregnancy is paramount and the patient needs to be informed that almost one in five philtres remain in situ with subsequent increased risk of post thrombotic syndrome.

\section{PM.81 THE IMPACT OF AN OBSTETRIC ANAESTHETIC ANTENATAL CLINIC FOR THE MORBIDLY OBESE, A RETROSPECTIVE STUDY}

doi:10.1136/archdischild-2013-303966.162

A Patience, MWJ MacDougall. Royal Victoria Infirmary, Newcastle Upon Tyne, UK

The CMACE/RCOG joint guideline recommend in their management of women with obesity in pregnancy guideline that pregnant women with a booking BMI > 40 should have an antenatal consultation with an obstetric anaesthetist. We compare the pregnancy outcome data in our morbidly obese population from both before and after the introduction of our specialist obesity obstetric anaesthetic clinic.

We performed a retrospective audit, comparing pregnancy outcomes in the first six months of 2009 pre-clinic, and the first six months of 2011 post clinic establishment, in the morbidly obese, looking at; anaesthetic type, estimated blood loss, cord $\mathrm{pH}<7.1$, cord $\mathrm{pH}<7.0$, and APGARs at 1, 5 and 10 minutes. The results are reviewed in the context of mode of delivery, induction, preterm delivery and birth weight.

Results The type of anaesthetic for elective section was 12/12 $(100 \%)$ spinal in 2009 v $10 / 11(90.9 \%)$ spinal $+1 / 11(9.1 \%)$ epidural in 2011. For emergency section the type of anaesthetic was $3 / 12$ $(25 \%)$ epidural $+9 / 12(75 \%)$ spinal in 2009 v $10 / 15(66.7 \%)$ epidural with one conversion to general anaesthetic $(6.7 \%)+5 / 15(33.3 \%)$ spinal in 2011. Estimated blood loss in 2011 appeared higher than in 2009 , but was not significantly so. Comparing EBL in 2009 v 2011; $23 / 46(50 \%)$ v35/70 (50\%) had $<500 \mathrm{ml}, 20 / 46$ (43.3\%) v25/70 $(35.7 \%)$ had $500-999 \mathrm{ml}, 1 / 46(2.1 \%)$ v $4 / 70$ (5.7\%) lost $1000-$ $1499 \mathrm{ml}, 2 / 46(4.3 \%)$ v 5/70 (7.1\%) lost $>1500 \mathrm{ml}$. Cord $\mathrm{pH}$ below 7.0 occurred in $1 / 46(2.1 \%)$ in 2009 v $1 / 70(1.4 \%)$ in 2011 . APGARS below 8 at 1,5 and 10 minutes were $3 / 46(6.5 \%), 1 / 46(2.1 \%)$ and $1 / 46(2.1 \%)$ respectively in 2009 and $13 / 70$ (18.6\%), 2/70 (2.8\%) and $0 / 70(0 \%)$ respectively in 2011 .

There were no obvious improvements in our outcome measures following the introduction of a consultant obstetric anaesthetist clinic for those with morbid obesity.

\section{PM.82 ACUTE HYPONATRAEMIA IN LABOUR - THE OBSTETRIC MARATHON?}

doi:10.1136/archdischild-2013-303966.163

P Sokhal, J Cook, S Robinson, L Lakasing. St Mary's Hospital, Imperial College Healthcare, London, UK

Maternal hyponatraemia during labour can affect both mother and baby. As a result, standard intrapartum care includes administration of oxytocin in sodium-containing fluids, limitation of oral intake and use of isotonic sports drinks. There is no strict guidance on best practise and local protocols vary.

We present a case report of acute severe hyponatraemia following spontaneous vaginal delivery at $38^{+5}$ weeks gestation in a 34-year-old primiparous woman. This previously well woman spent 4.5 hours in the birthing pool and drank approximately 6 litres of water/lucozade in that time. After delivery, she suffered a seizure and acute confusion. The plasma sodium was $117 \mathrm{mmol} / \mathrm{L}$ (135$145 \mathrm{mmol} / \mathrm{l})$. This was corrected with hypertonic saline to 130 $\mathrm{mol} / \mathrm{L}$ within 4 hours. She was admitted to intensive care and required sedation and ventilation. Endocrine investigations revealed no underlying cause. Differential diagnoses included atypical eclampsia and posterior-reversible encephalopathy syndrome. She was discharged on day 6 with a Mini-mental State Examination score of $30 / 30$

Isotonic drinks prevent urinary ketosis, maintain plasma glucose and electrolytes, thereby preventing the 'starvation effect' of labour, also seen in marathon runners. Review of the literature relating to the effect of water immersion and oral fluid intake on plasma sodium levels during labour suggests that a 40-minute bath may cause increased naturiesis and plasma volume expansion. Water tolerance appears diminished in labour and thus intoxication may be possible with relatively moderate volumes.

We recommend that in labour a) women do not drink excessively, b) hypotonic fluid administration is avoided, c) fluid-balance charts become mandatory.

\section{PM.83 TESTING FOR FETOMATERNAL HAEMORRHAGE BY ACID ELUTION CAN YIELD FALSE POSITIVE RESULTS IN THE PRESENCE OF ELEVATED MATERNAL FETAL HAEMOGLOBIN}

doi:10.1136/archdischild-2013-303966.164

A Doyle, J Donnelly, S Campbell, D Murphy, D Corcoran, B Kumpel, FD Ni Ainle. Rotunda Hospital, Rotunda, Ireland

Appropriate testing for fetomaternal haemorrhage (FMH) is critical in the prevention of morbidity and mortality due to haemolytic disease of the fetus and newborn (HDFN) in $\mathrm{RhD}$ negative women. The Kleihauer or acid elution (AE) test is widely used to assess the size of fetomaternal haemorrhage and to determine whether sufficient Anti-D immunoglobulin has been administered to prevent HDFN. This test is based on the principle that adult haemoglobin $(\mathrm{HbA})$ is eluted from red cells in an acidic solution while the fetal haemoglobin $(\mathrm{HbF})$ is not. However, in the presence of an elevated level of $\mathrm{HbF}$ of maternal origin, the AE test may be "positive" in the absence of a true FMH. We report two cases in which this situation arose antenatally, leading to difficulties in clinical interpretation. In both cases, specialised flow cytometry revealed the presence of elevated levels of $\mathrm{HbF}$ of maternal origin. While awaiting completion of specialised investigations, Anti-D was administered but was later found to have been unnecessary. Early awareness of the possibility of elevated maternal F cells ensures that samples can be sent to appropriate reference laboratories early to limit unnecessary Anti-D administration. The true prevalence of "false positive" AE tests due to elevated maternal $\mathrm{HbF}$ is unknown and is the subject of ongoing work in our laboratory, but should be considered in the differential diagnosis of an $\mathrm{AE}$ test remaining positive despite appropriate Anti-D administration.

\section{PM.84 THE ASSOCIATION BETWEEN SEVERITY OF IMPAIRED GLUCOSE TOLERANCE IN GESTATIONAL DIABETES WITH AGE, BMI AND ETHNICITY}

doi:10.1136/archdischild-2013-303966.165

B Jones, S Balaji, C Cotzias. The West Middlesex University Hospital, Isleworth, UK 